\title{
Mining yeast in silico unearths a golden nugget for mitochondrial biology
}

\author{
Robert L. Nussbaum \\ Genetic Diseases Research Branch, National Human Genome Research Institute, Bethesda, Maryland, USA.
}

\begin{abstract}
NADH:ubiquinone oxidoreductase (complex I) of the electron transport chain is a multimeric mitochondrial enzyme of approximately $1000 \mathrm{kDa}$ consisting of 46 different proteins encoded by both the mitochondrial and nuclear genomes. Little is known about the cellular mechanisms and protein chaperones that guide its assembly. In this issue of the JCI, Ogilvie et al. use genomic sequence data to compare the proteins produced by yeasts with and without complex $I$ in order to generate a list of proteins whose human orthologs might serve as complex I assembly proteins (see the related article beginning on page 2784). The gene encoding one of these candidate proteins, $B 17.2 L$, was found to harbor a nonsense mutation in one of 28 patients with a deficiency of complex I. B17.2L associated with subcomplexes that are seen when complex I assembly is incomplete. The research described here combines clever model organism genomics and bioinformatics with sophisticated human molecular and biochemical genetics to identify the first mammalian protein required for the normal assembly of complex I.
\end{abstract}

\section{Complex I of the mitochondrial respiratory chain}

Of all the components of the electron transport chain, NADH:ubiquinone oxidoreductase (complex I) is by far the biggest and contains the largest number of structural proteins (1). Complex I contains the mitochondrial NADH-dehydrogenase (EC 1.6.99.3) activity responsible for accepting electrons from $\mathrm{NADH}$ and passing them on via ubiquinone to complex III while pumping 4 protons across the inner membrane, thereby contributing to the electrochemical ion gradient across the inner mitochondrial membrane that powers the synthesis of ATP (2) (Figure 1). Based on studies of bovine heart mitochondria, complex I is a very large multisubunit structure, approximately $1000 \mathrm{kDa}$ in size and made up of at least 46 subunits (3), of which 39 are nuclear encoded and 7 are encoded by the mitochondrial genome. According to low-resolution electron micrographs, complex I is an L-shaped heteromultimer (1). A relatively hydrophilic matrix arm extends into the interior of the mitochondria and is connected to a more hydrophobic seg-

Nonstandard abbreviations used: BN-PAGE, blue native PAGE; complex I, NADH:ubiquinone oxidoreductase.

Conflict of interest: The author has declared that no conflict of interest exists.

Citation for this article: J. Clin. Invest. 115:2689-2691 (2005). doi:10.1172/JCI26625. ment located within the inner mitochondrial membrane (4) (Figure 1). The precise molecular mechanisms that couple electron transfer, ubiquinone reduction, and proton pumping still need to be elucidated (2).

As a complicated multisubunit structure of 46 proteins, complex I must be assembled from smaller subcomplexes, which are themselves assembled from smaller intermediates. The existence of subcomplex intermediates in complex I assembly is supported by the observation that $2 \mathrm{D}$ blue native PAGE (BN-PAGE) of complex I from muscle mitochondria obtained from 4 patients with complex I deficiency, 1 with a known mutation in the mitochondrial MTND2 gene and the others carrying mutations in undefined nuclear-encoded genes, revealed the existence of several common subcomplexes of varying sizes (5). A different but reproducible subunit expression pattern was seen in an analysis of 2D BN-PAGE of subcomplex assembly occurring after release of a block in mitochondrial protein translation induced by doxycycline (6). The complicated process of assembling such a large multimeric complex most likely needs to be guided by cellular machinery (6). Studies in the aerobic fungus Neurospora crassa have identified 2 of the proteins required to assemble the segment of complex I that is located in the inner mitochondrial membrane from 2 smaller subcomplexes (7).
Researchers have not yet been able to bring the full power of genetic analysis to elucidating the structure and function of complex I because the usual eukaryotic workhorse for such approaches, the yeast Saccharomyces cerevisiae, lacks complex I. Although there has been a lot progress in using prokaryotes or eukaryotic fungi such as $N$. crassa as alternative model systems (7), the pace of progress seems to now be accelerating through the use of Yarrowia lipolytica, an obligate aerobic yeast and a powerful new model organism for studies of complex I structure and function (1). Complex I from Y. lipolytica appears to be very similar to mammalian complex I in structure and composition, and its identification has facilitated the usual opportunities for genetic manipulation in yeast, such as mutagenesis screens for functional mutants, facile site-directed mutagenesis, and expression of tagged proteins suitable for affinity chromatography and proteomic analysis (8).

\section{Disorders of oxidative phosphorylation in humans}

Human diseases caused by defects in oxidative phosphorylation are rare (approximately 1 per 10,000 live births) but often take the form of devastating neurological conditions (9). Symptoms can vary from fatal lactic acidosis in the neonate to mental and physical retardation with cardiomyopathy, skeletal myopathy, and hepatic failure in childhood, to acute painless loss of vision (Leber hereditary optic neuropathy) in young adults, to a form of Parkinson disease later in life. One-third of the defects in the electron transport chain that cause genetic oxidative phosphorylation diseases occur in complex I $(9,10)$. Only a minority of the molecular abnormalities that cause complex I deficiency are known. Laboratories that do extensive molecular diagnostic analysis for complex I deficiency report that only approximately $20-25 \%$ of such patients have homoplasmic or heteroplasmic mutations in 1 of 4 mitochondrial-encoded complex I subunit genes; another $20-25 \%$ are the result of mutations in 1 of 9 nuclear-encoded complex I 


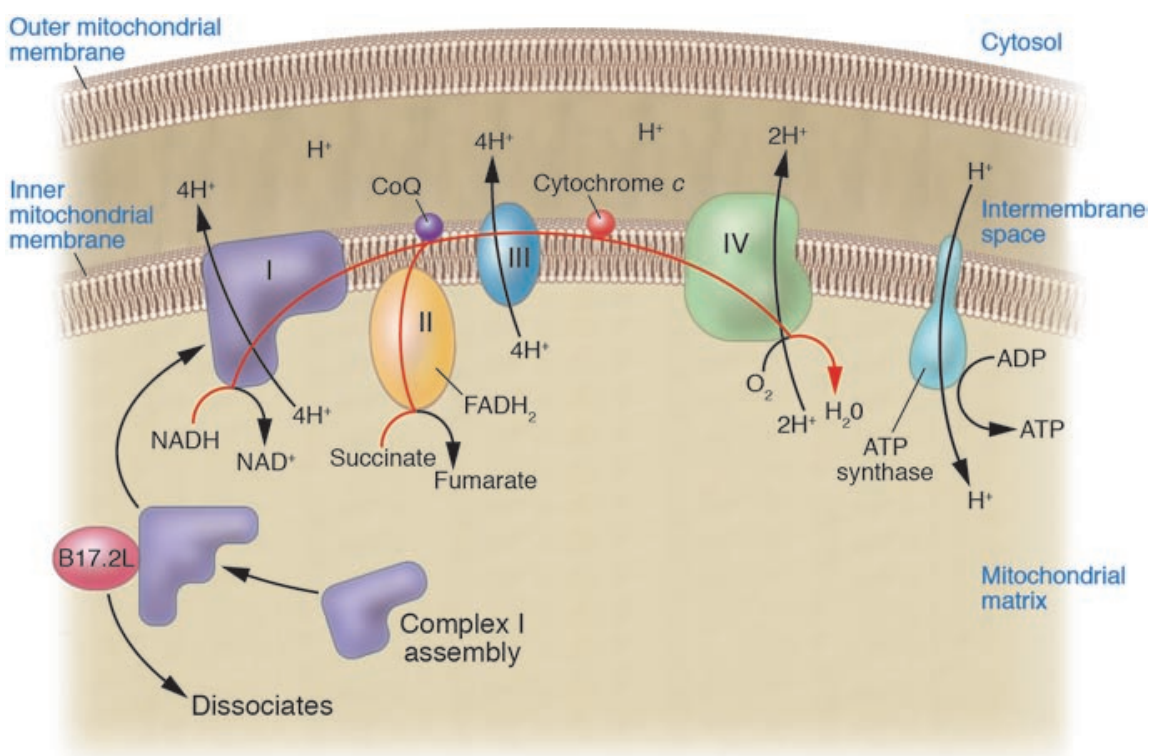

\section{Figure 1}

Schematic diagram of complexes I through IV of the electron transport chain and ATP synthase. The red line traces the path of electrons as they enter and move along the electron transport chain. Complex I is shown at the far left as an L-shaped structure with one portion extending down into the mitochondrial matrix and the other portion embedded in the inner mitochondrial membrane. Ogilvie and colleagues used bioinformatics to perform a virtual whole genome subtraction of yeasts with or without complex I to find candidate complex I assembly factors, identified the human orthologs of these proteins, and showed that one of these orthologs, B17.2L, carried a null mutation in a patient with complex I deficiency (17). The authors also demonstrate that $\mathrm{B} 17.2 \mathrm{~L}$ - the first identified mammalian protein required for the normal assembly of human complex I - associates with an immature subcomplex of complex I (shown schematically) and facilitates complex I assembly. $\mathrm{FADH}_{2}$, reduced flavin-adenine dinucleotide; $\mathrm{CoQ}$, coenzyme $\mathrm{Q}$ (also known as ubiquinone).

structural subunit genes $(9,11-13)$. The molecular defects in the remaining $50-60 \%$ of patients with deficiencies in complex I, but without obvious mutations in genes encoding complex I structural subunits, still remain largely undetermined. It seems a very reasonable supposition that some of these complex I defects without structural subunit mutations are caused by defects in auxiliary proteins required for multimer assembly, as has already been demonstrated in some patients with severe encephalopathy and failure of other organ systems due to mutations in genes such as SURF1 and SCO2 that affect complex IV assembly (14) or mutations in $B C S 1 L$ affecting complex III assembly (15). Of the 2 assembly proteins that have been shown to be required for $N$. crassa complex I assembly, 1 has a human ortholog; however, no defects in that gene have been found in patients with complex I deficiency (16). In this issue of the JCI, Ogilvie et al. report the first human protein required for assembly of human complex I (17).

Ogilvie et al. (17) report a female child, born to a normal, nonconsanguineous cou- ple, who developed progressive neurological disease affecting many portions of her central nervous system beginning around 1 year of age and suffered relentless neurological deterioration until her death at 13.5 years of age. Her disease was associated with elevation of cerebral spinal fluid lactate levels and a deficiency of complex I enzyme activity in muscle mitochondria (approximately $38 \%$ of control complex I activity) and cultured fibroblasts (less than $20 \%$ of control complex I activity). Taking a clever bioinformatics approach in the appropriate model organisms, these researchers carried out a subtraction in silico of genes found in $Y$. lipolytica and another aerobic yeast with a complex I, Debaryomyces hansenii, but not in other yeasts that lack a complex I, and used the resulting protein sequences to search for human orthologs containing mitochondrial targeting sequences. Their analysis ultimately yielded 14 genes, 1 of which was $B 17.2 L$, a paralog of B17.2, which encodes a known structural subunit in the matrix arm of human complex I (18). They sequenced $B 17.2 \mathrm{~L}$ in 28 patients with complex I deficiency and found 1 patient, the child described above, who appeared to be homozygous for a nonsense mutation (C182T) in exon 2 of B17.2L that caused premature termination of translation. Her mother was heterozygous for this mutation, but the mutation was not found in her father, suggesting that he was likely to be heterozygous for a deletion allele that the proband inherited from him as her paternal allele. The functional complex I deficiency and defective assembly in this patient, as determined by enzyme assay and 2D BN-PAGE, was corrected by transduction with a vector expressing B17.2L cDNA. Finally, Ogilvie et al. demonstrated that the $\mathrm{B} 17.2 \mathrm{~L}$ protein associates with a particular $830-k D a$ subcomplex of complex I that accumulates in a variety of patients with mutations in genes encoding structural components of complex I, but not with the normal intact complex I itself. Based on these data, the authors concluded that B17.2L is a component of the cellular machinery that is involved in the assembly of complex I without being a part of the mature complex I itself and that loss of function of this protein leads to complex I deficiency (Figure 1).

Humans stand at the opposite end of the spectrum from yeast in terms of serving as an easily manipulated genetic system. However, the study of human genetics has much to offer, not only because of the direct involvement with human disease, but also because of the depth of phenotypic richness and the locus and allelic heterogeneity that human genetic disease provides. Indeed, one of the more striking themes of modern molecular genetics has been how progress in understanding fundamental biological processes has come time and again from the marriage of model organism research with careful human genetic studies. The research reported here by Ogilvie and colleagues (17) is an excellent example of just such a successful marriage.

\section{Acknowledgments}

The author is supported by the Intramural Research Program of the National Human Genome Research Institute, NIH.

Address correspondence to: Robert L. Nussbaum, Genetic Diseases Research Branch, National Human Genome Research Institute, 49 Convent Drive, Room 4A72, Bethesda, Maryland 20892, USA. Phone: (301) 402-2039; Fax: (301) 402-2170; E-mail: rlnuss@mail.nih.gov. 
1. Djafarzadeh, R., et al. 2000. Biophysical and structural characterization of proton-translocating NADH-dehydrogenase (complex I) from the strictly aerobic yeast Yarrowia lipolytica. Biochim. Biophys. Acta. 1459:230-238.

2. Hirst, J. 2005. Energy transduction by respiratory complex I-an evaluation of current knowledge. Biochem. Soc. Trans. 33:525-529.

3. Carroll, J., Fearnley, I.M., Shannon, R.J., Hirst, J., and Walker, J.E. 2003. Analysis of the subunit composition of complex I from bovine heart mitochondria. Mol. Cell Proteomics. 2:117-126.

4. Grigorieff, N. 1998. Three-dimensional structure of bovine NADH:ubiquinone oxidoreductase (complex I) at $22 \mathrm{~A}$ in ice. J. Mol. Biol. 277:1033-1046.

5. Antonicka, H., et al. 2003. Identification and characterization of a common set of complex I assembly intermediates in mitochondria from patients with complex I deficiency. J. Biol. Chem. 278:43081-43088.

6. Ugalde, C., et al. 2004. Human mitochondrial complex I assembles through the combination of evolutionary conserved modules: a framework to interpret complex I deficiencies. Hum. Mol. Genet. 13:2461-2472.

7. Schulte, U. 2001. Biogenesis of respiratory complex I. J. Bioenerg. Biomembr. 33:205-212.

8. Brandt, U., et al. 2005. Structure-function relationships in mitochondrial complex I of the strictly aerobic yeast Yarrowia lipolytica. Biochem. Soc. Trans. 33:840-844.

9. Triepels, R.H., Van Den Heuvel, L.P., Trijbels, J.M., and Smeitink, J.A. 2001. Respiratory chain complex I deficiency. Am. J. Med. Genet. 106:37-45.

10. von Kleist-Retzow, J.C., et al. 1998. A high rate (20\%$30 \%$ ) of parental consanguinity in cytochrome-oxidase deficiency. Am. J. Hum. Genet. 63:428-435.

11. OMIM - Online Mendelian Inheritance in Man. http://www.ncbi.nlm.nih.gov/entrez/query. fcgi? db=OMIM.

12. Robinson, B.H. 1998. Human complex I deficiency: clinical spectrum and involvement of oxygen free radicals in the pathogenicity of the defect. Biochim. Biophys. Acta. 1364:271-286.

13. Benit, P., et al. 2004. Mutant NDUFS3 subunit of mitochondrial complex I causes Leigh syndrome. J. Med. Genet. 41:14-17.

14. Rotig, A., et al. 2004. Molecular diagnostics of mitochondrial disorders. Biochim. Biophys. Acta. 1659:129-135

15. de Lonlay, P., et al. 2001. A mutant mitochondrial respiratory chain assembly protein causes complex III deficiency in patients with tubulopathy, encephalopathy and liver failure. Nat. Genet. 29:57-60.

16. Janssen, R., Smeitink, J., Smeets, R., and van Den Heuvel, L. 2002. CIA30 complex I assembly factor: a candidate for human complex I deficiency? Hum. Genet. 110:264-270.

17. Ogilvie, I., Kennaway, N.G., and Shoubridge, E.A. 2005. A molecular chaperone for mitochondrial complex I assembly is mutated in a progressive encephalopathy. J. Clin. Invest. 115:2784-2792. doi:10.1172/JCI26020.

18. Smeitink, J., et al. 1998. Molecular characterization and mutational analysis of the human B17 subunit of the mitochondrial respiratory chain complex I. Hum. Genet. 103:245-250.

\title{
PDGF signaling in pulmonary arterial hypertension
}

\author{
Robyn J. Barst
}

New York Presbyterian Pulmonary Hypertension Center, Columbia University College of Physicians \& Surgeons, New York, New York, USA.

\begin{abstract}
The pathobiology of pulmonary arterial hypertension (PAH) includes endothelial cell dysfunction and proliferation and migration of VSMCs. As PDGF has been implicated in these processes, Schermuly et al. hypothesized that altered PDGF signaling may be involved in the vascular remodeling observed in PAH. To explore this notion further, the authors evaluated the effects of the PDGF receptor inhibitor STI571 in 2 different animal models of pulmonary hypertension (see the related article beginning on page 2811). In both models, after development of pulmonary vascular disease, administration of STI571 reversed pulmonary vascular changes. These studies provide preclinical proof of concept for the clinical development of a PDGF inhibitor as a targeted therapy for PAH patients.
\end{abstract}

Pulmonary arterial hypertension (PAH), a disorder limited to the pulmonary circulation, is characterized by pulmonary vascular obstruction and variable pulmonary vasoconstriction leading to increased pulmonary vascular resistance and death. PAH can be idiopathic, or unexplained (formerly termed primary pulmonary hypertension); PAH can also occur in association with connective tissue diseases, HIV infection, congenital heart disease, portal hypertension, and appetite suppressant exposure. Idiopathic PAH occurs more often in women than in men,

Nonstandard abbreviations used: BMP, bone morphogenetic protein; $\mathrm{PAH}$, pulmonary arterial hypertension.

Conflict of interest: The author serves as a consultant to Actelion Ltd., CoTherix Inc., Encysive Pharmaceuticals Inc., INO Therapeutics Inc., Mondo Developments, Myogen Inc., GlaxoSmithKline Inc., Pfizer Inc., and United Therapeutics Corp.

Citation for this article: J. Clin. Invest. 115:2691-2694 (2005). doi:10.1172/JCI26593. with a median survival of 2.8 years if untreated. The mean age at diagnosis is 35 years, i.e., it occurs most often in young adults with no other comorbid conditions. Although current treatment options have markedly improved overall quality of life and survival in PAH, 5-year survival remains at 50\% for this devastating disease. As discussed below, over the past 2 decades, we have learned a great deal about the pathobiology of PAH; however, we still do not know what initiates this disease with its subsequent progressive pulmonary vascular obstruction.

PAH has a multifactorial pathobiology. Vasoconstriction, remodeling of the pulmonary vessel wall, and thrombosis contribute to increased pulmonary vascular resistance in PAH. The process of pulmonary vascular remodeling involves all layers of the vessel wall and is complicated by cellular heterogeneity within each compartment of the pulmonary arterial wall (Fig- ure 1). Indeed, each cell type (endothelial, smooth muscle, and fibroblast), as well as inflammatory cells and platelets, may play a significant role in PAH. Endothelial dysfunction is considered a key element in the pathobiology of $\mathrm{PAH}$, with increased levels of endothelin occurring concomitantly with decreased $\mathrm{NO}$ and prostacyclin levels (1). Although it remains unclear whether excessive vasoconstriction is associated with the endothelial dysfunction, many of the perturbations associated with the endothelial dysfunction promote vascular remodeling in addition to increasing pulmonary vascular tone. Prostacyclin, $\mathrm{NO}$, endothelin, angiopoietin I, serotonin, cytokines, chemokines, and members of the TGF- $\beta$ superfamily have all been implicated in the pathobiology of PAH. As a result, 3 of the currently approved therapeutic modalities for the treatment of $\mathrm{PAH}$ - prostacyclins, phosphodiesterase inhibitors, and endothelin receptor antagonists (2-4) - target prostacyclin and NO deficiencies and increased endothelin levels, respectively, in $\mathrm{PAH}$ patients.

Distal extension of smooth muscle cells into small, peripheral, normally nonmuscular pulmonary arterioles is a hallmark of PAH. However, the processes causing this migration as well as the formation of a layer of microfibroblasts and extracellular matrix (termed the neointima) between the endothelial cells and the internal elas- 